\title{
Analysis of Internal Transport Barrier Formation in Tokamak Reactor Plasmas
}

\author{
Yoshihito HORI, Kozo YAMAZAKI, Tetsutarou OISHI, Hideki ARIMOTO and Tatsuo SHOJI \\ Nagoya University, Furo-cho, Chikusaku, Nagoya 464-8603, Japan
}

(Received 9 December 2009 / Accepted 4 April 2010)

\begin{abstract}
Since a high performance operation is needed in the future fusion reactor, it is expected that the operation with Internal Transport Barrier (ITB) is utilized to improve core plasma confinement in the reversed magnetic shear. In this study, firstly, the simulation results are compared with the experimental result, and the validity of the model is checked. Secondly, the effects of the magnetic shear and the pellet injection on ITB formation are simulated in tokamak reactor using the Toroidal Transport Analysis Linkage code TOTAL. In this simulation, the ITB formation is confirmed in the condition that magnetic shear is nearly zero and the density gradient exists, and it found that the reversed magnetic shear configuration is effective in the operation with ITB.
\end{abstract}

(C) 2010 The Japan Society of Plasma Science and Nuclear Fusion Research

Keywords: transport simulation, internal transport barrier, magnetic shear, pellet injection, tokamak reactor

DOI: $10.1585 /$ pfr.5.S2034

\section{Introduction}

Fusion power output and required external heating power in fusion reactors strongly depend on the radial profile of plasma temperature and density. Recently the reactor system analysis has been done, and the transport simulation studies have been carried out focusing on the dependence of fusion power on the density profile. These studies have been revealed that it is important to control core plasma density profile to optimize reactor operations. In general, higher output power is obtained by making temperature and density profiles peaked, and an optimized operation becomes possible. On the other hand, it is considered that an advanced operation scenario using reversed magnetic shear mode and higher poloidal beta mode with internal transport barrier (ITB) will be needed for confinement improvement and higher bootstrap current fraction in future reactors. It is expected that reliable operation with ITB enables an optimized operation to improve burning plasma confinement. When the ITB is formed in the reversed shear, it brings good confinement rather than peaked pressure profile, so that we examine the relationship between the ITB formation and the magnetic shear.

The transport simulations have been carried out focusing on the ITB formation in tokamak and helical plasmas. The ITB model based on Bohm and GyroBohm-like transport with $E \times B$ shear flow effects has already been compared with the JET experimental ITB in tokamak systems [1] and in helical system compared with LHD [2]. This model is introduced into the toroidal transport linkage TOTAL code [3,4], and is applied to the 1-dimensional (1-D) ITB formation simulation of 2-D equilibrium tokamk plasmas and 3-D equilibrium helical.

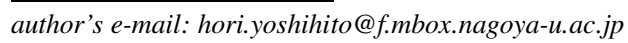

Section 2 will describe the details of the transport models and pellet injection model included in the TOTAL code, and simulation results will be shown in section 3 . The conclusion will be given in section 4 .

\section{Numerical Model}

\subsection{Transport model description}

The Bohm and GyroBohm mixed transport model with the $E \times B$ shear flow effect has already been compared with the helical and tokamak experimental ITBs $[1,2]$. The most widely accepted explanation for the ITB formation relies on the suppression of ITG turbulence due to $E \times B$ shear flow. The suppression of the turbulence might occur when the $E \times B$ flow shearing rate $\omega_{\mathrm{E} \times \mathrm{B}}$ exceeds the ITG linear growth rate $\gamma_{\mathrm{ITG}}$. The shearing rate $\omega_{\mathrm{E} \times \mathrm{B}}$ is defined as $[5,6]$

$$
\omega_{\mathrm{E} \times \mathrm{B}}=\left(\frac{\Delta \psi_{0}}{\Delta \phi_{0}}\right)\left|\frac{\partial^{2} \Phi_{0}(\psi)}{\partial \psi^{2}}\right| \cong\left|\frac{R B_{\theta}}{B_{\phi}} \frac{\partial}{\partial r}\left(\frac{E_{r}}{R B_{\theta}}\right)\right|,
$$

where $\Delta \psi_{0}$ and $\Delta \phi_{0}$ are the correlation lengths of the ambient turbulence in the radial and toroidal direction, and $\Phi_{0}$, $E_{r}, B_{\theta}$ and $B_{\phi}$ are the equilibrium electrostatic potential, the radial electric field, the poloidal and toroidal magnetic field, respectively. In tokamaks, the radial electric field $E_{r}$ is not easily measured directly, so that $E_{r}$ can be calculated from the plasma radial force balance equation under the assumption that the poloidal velocities can be expressed according to the neoclassical theory $[7,8]$. However in this paper, $E_{r}$ is described simply as

$$
\frac{\mathrm{d} E_{r}}{\mathrm{~d} r} \cong-\frac{1}{e n_{\mathrm{i}}^{2}} \frac{\mathrm{d} n_{\mathrm{i}}}{\mathrm{d} r} \frac{\mathrm{d} p_{\mathrm{i}}}{\mathrm{d} r}
$$

in the H-mode condition [9], where $n_{\mathrm{i}}$ and $p_{\mathrm{i}}$ are ion density and ion pressure, respectively. The ITG growth rate 
$\gamma_{\mathrm{ITG}}$ is defined as [10]

$$
\gamma_{\mathrm{ITG}}=\frac{\left(\eta_{\mathrm{i}}-2 / 3\right)^{1 / 2}|s| c_{\mathrm{i}}}{q R},
$$

where $\eta_{\mathrm{i}}=L_{\mathrm{n}} / L_{\mathrm{T}}, c_{\mathrm{i}}=\sqrt{T_{\mathrm{i}} / m_{\mathrm{i}}}$, and $s$ is the magnetic shear defined as

$$
s=\frac{r}{q}\left(\frac{\mathrm{d} q}{\mathrm{~d} r}\right) .
$$

Most theoretical studies based on the $E \times B$ shear stabilization adopt a thermal diffusion coefficient $\chi$ in the form

$$
\begin{aligned}
& \chi_{\mathrm{e}, \mathrm{i}}=\chi_{\text {neoclassical }}+\chi_{\text {anomalous }}, \\
& \chi_{\text {anomalous }}=\left(\alpha_{1} \times \chi_{\text {Gyrobohm }}+\alpha_{2} \times \chi_{\text {Bohm }}\right) \\
& \times F\left(\omega_{\mathrm{E} \times \mathrm{B}} / \gamma_{\mathrm{ITG}}\right),
\end{aligned}
$$

where

$$
F\left(\omega_{\mathrm{E} \times \mathrm{B}} / \gamma_{\mathrm{ITG}}\right)=\frac{1}{1+\left\{\tau \times\left(\omega_{\mathrm{E} \times \mathrm{B}} / \gamma_{\mathrm{ITG}}\right)\right\}^{\gamma}} .
$$

The coefficient $\chi_{\text {neoclassical }}$ is the neoclassical part of thermal diffusion coefficient, and $\chi_{\text {anomalous }}$ is the anomalous part described as the Bohm and GyroBohm mixed transport model $[1,2]$. And particle diffusion coefficient $D$ is assumed as $D=\chi_{\mathrm{e}, \mathrm{i}} / C_{\mathrm{ano}}$, in this paper $C_{\text {ano }}=3, \tau=2.0$, and $\gamma=4.0$ (tokamak).

\subsection{Pellet injection}

The pellet injection is described as the process of the pellet ablation. We simulate the injection with the pellet penetration model with the ablation model. There are a few models which satisfactorily describe the pellet ablation and give similar results [11]. So, we use here the most popular one: the neutral gas shielding (NGS) model [12]. This ablation rate by the scaling is described as

$$
\begin{array}{r}
\frac{\mathrm{d} N}{\mathrm{~d} x}=1.12 \times 10^{16} n_{\mathrm{e}}^{0.333} T_{\mathrm{e}}^{1.64} r_{\mathrm{p}}^{1.333} M_{\mathrm{i}}^{-0.333} V_{\mathrm{p}}^{-1} \\
{[\text { atoms } / \mathrm{m}],}
\end{array}
$$

where $n_{\mathrm{e}}, T_{\mathrm{e}}, r_{\mathrm{p}}, M_{\mathrm{i}}$ and $V_{\mathrm{p}}$ are electron density, electron temperature, pellet radius, particle mass in pellet and injection velocity, respectively.

The pellet injection makes the local density gradient quickly, and the electric field gradient is formed. Because the shear flow $\omega_{\mathrm{E} \times \mathrm{B}}$ grows in the reversed shear region, the stabilization requirement $\omega_{\mathrm{E} \times \mathrm{B}}>\gamma_{\mathrm{ITG}}$ can be satisfied, which leads to the improvement of transport coefficients.

\section{Simulation Results}

\subsection{The comparison with experimental data}

Two typical JT-60U parameters used in this simulation are shown in Table 1 . Figure 1 (a) (d) shows the radial profiles of experimental data in JT-60U. These experimental data are based on The International Multi-Tokamak Confinement Profile Database. In Fig. 1, left figures show
Table 1 Typical JT-60U experimetnal parameters.

\begin{tabular}{cccccccc}
\hline Device & $\begin{array}{c}\text { Shot } \\
\text { number }\end{array}$ & $\begin{array}{c}\mathrm{R}_{\text {maj }} \\
{[\mathrm{m}]}\end{array}$ & $\begin{array}{c}\mathrm{a}_{\min } \\
{[\mathrm{m}]}\end{array}$ & $\mathrm{Bt}[\mathrm{T}]$ & $\delta$ & $\kappa$ & Shear \\
\hline JT-60U & $\# 21811$ & 4.424 & 0.944 & 4.008 & 0.185 & 1.43 & Normal \\
JT-60U & $\# 32423$ & 3.368 & 0.7949 & 3.836 & 0.2319 & 1.597 & Reversed \\
\hline
\end{tabular}

* The International Multi-Tokamak Confinement Profile Database
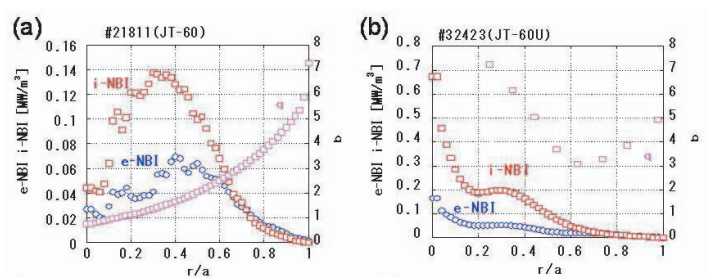

(c)
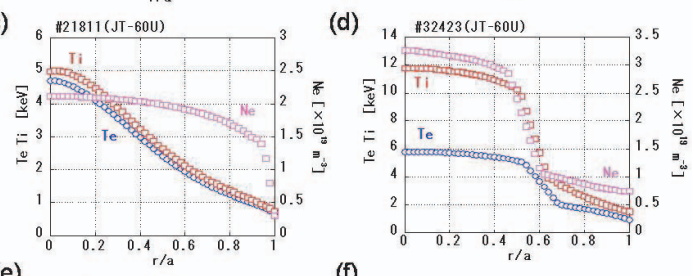

(e)
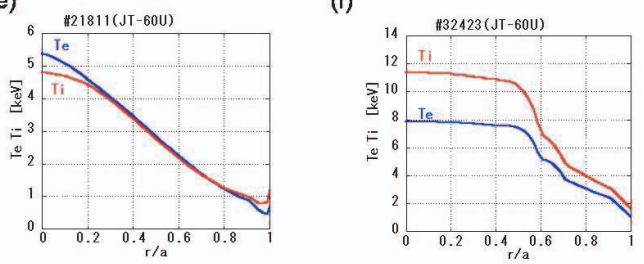

Fig. 1 The experimental data (JT-60U) and simulation result. Left figures denote the positive shear case, and right figures are the reversed shear case. The upper figures (a) (d) show the radial profiles of NBI heating, safety factor, electron density and temperature profiles in JT$60 \mathrm{U}$, and the lower figures (e) and (f) show the temperature profiles in simulation.

the positive magnetic shear case without ITB, and right figures are the reversed case with ITB.

The temperature profiles were compared in the condition of fixing NBI heating, safety factor and electron density profiles. These simulation results are shown in Fig. 1 (e) and (f). It is seen that correspondence of temperature profiles between the experimental data and simulation results in positive magnetic shear, and the ITB's foot position and shape of temperature profiles are corresponding well in the reversed shear. Therefore, we can confirm the reliability of the simulation model both in the positive and reversed magnetic shear.

\subsection{ITB formation by difference of magnetic shear}

In the previous subsection, we show that the reliability of the simulation model is confirmed in both normal and reversed shear cases. In this subsection, we compared the radial profiles simulated in the cases of the three different shear distributions. The cases are positive, weak reversed and strong reversed magnetic shear, and they are quite dif- 
(a)

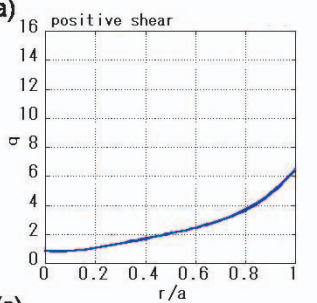

(c)

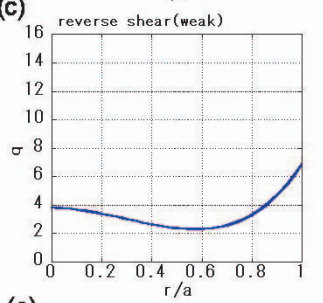

(e)

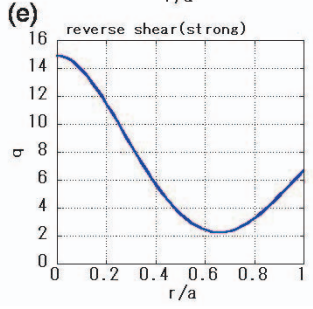

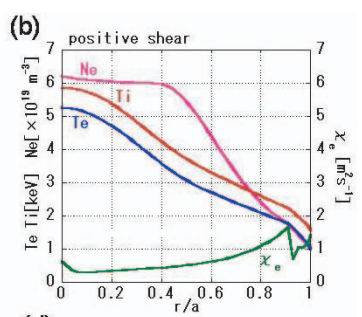

(d)
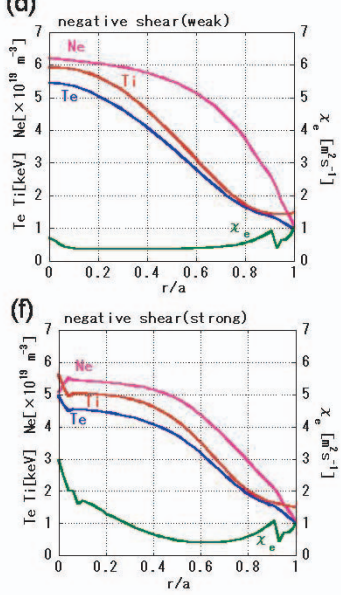

Fig. 2 The radial profiles in magnetic shear difference. Left figures correspond safety factor, and right figures are the radial profiles of temperature, electron density and transport coefficient. Figure (a) and (b) show the positive shear case, figure (c) and (d) are the weak reversed shear case, and figure (e) and (f) are the strong reversed shear case.

ferent profiles with each other. The simulated operation is under the condition that density profile is almost equal in the JT-60U like reactor. These simulation results are shown in Fig. 2.

As a result, in the reversed magnetic shear, a little reduction of transport coefficients is seen at the position where the magnetic shear is nearly zero, as compared to the positive one. But ITB cannot be seen in this condition. In the comparison between weak and strong of the reversed shear, it seems that the electron transport coefficients depend on the value of safety factor, and is not related to the absolute value of magnetic shear. So, it is considered that the strong reversed shear isn't needed at the point of transport coefficients.

It seems that the reversed shear is better than the positive one. In the case without ITB, a little improvement is seen, but the large improvement doesn't occur. It shows that the $E \times B$ shear term doesn't overcome the ITG unstable term only by the magnetic shear change in the present model.

\subsection{ITB formation with pellet injection}

In the previous subsection, we cannot find the profiles with ITB, so that we consider the case of pellet injection. The pellet injection causes the pressure gradient and the electric field gradient in the core plasma because
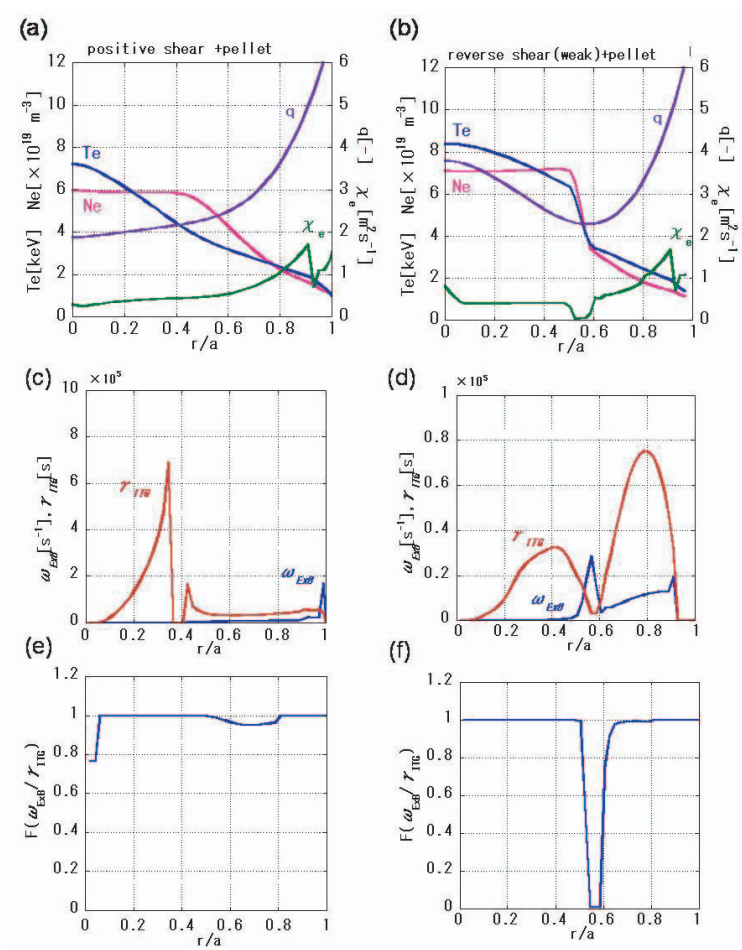

Fig. 3 Simulation results with the pellet injection. Left figures (a), (c) and (e) denote the positive shear case, and right figures (b), (d) and (f) correspond to the reversed shear case. The figures (a) and (b) show radial profiles of safety factor, electron temperature, density and transport coefficient.Figures (c), (d) show profiles of $\omega_{\mathrm{E} \times \mathrm{B}}$ and $\gamma_{\mathrm{ITG}}$, and (e), (f) are the transport reduction factor in eq. (6).

large electric field gradient grows the stable term $\omega_{\mathrm{E} \times \mathrm{B}}$. We assume the operation with the pellet injection which penetration length is $r / a \sim 0.4$ in the JT-60U like reactor. The simulation results are shown in Fig. 3 (a) is the positive magnetic shear case, and (b) is the reversed case. In Fig. 3 (c) and (d) show $\omega_{\mathrm{E} \times \mathrm{B}}, \gamma_{\mathrm{ITG}}$ and reduction factor in the case (a). These profiles in the case (b) are shown in figures (d) and (f).

Judging from the density and temperature profiles in the reversed magnetic shear, it is seen that ITB's foot exists at the position the magnetic shear is nearly zero: $r / a \sim 0.6$. The decrease in transport coefficients is seen locally only in the reversed one. It isn't made by the pellet injection because penetration length is deeper than the position where the magnetic shear is zero. So, it is considered that the pressure gradient brought by pellet injection makes the electric field gradient, and increases stable $E \times B$ shearing rate $\omega_{\mathrm{E} \times \mathrm{B}}$ larger than the unstable $\gamma_{\mathrm{ITG}}$ rate. Therefore, the stability condition is satisfied, and large transport improvement occurs at the position where the magnetic shear is nearly zero.

It is clear that the profile with ITB is better at the point of energy confinement improvement as shown in Fig. 3. Though the pellet injection is needed for ITB formation in 
the present model, the results show that the reversed magnetic shear configuration is required. So, it seems that the reversed shear is effective in the operation with ITB.

\section{Conclusion}

The effect of the magnetic shear difference on the ITB formation is simulated. In comparison with JT-60U experimental data, we confirm the reliability of the simulation model. In the case of gas puff fueling, a little reduction of transport coefficients is seen in the case of reversed magnetic shear, but the formation of ITB is not realized in the present time dependent simulation. In the case of pellet injection, ITB formation is confirmed in the reversed magnetic shear. However it cannot be formed in positive magnetic shear because the unstable ITG mode is not stabilized in the present model. Therefore, it is considered that the reversed magnetic shear is effective in the operation with ITB.
[1] T. Tala et al., Plasma Phys. Control. Fusion 43, 507 (2001).

[2] J. Garcia, K. Yamazaki, J. Dies and J. Izquierdo, Phys. Rev. Lett. 96, 105007 (2006).

[3] K. Yamazaki and T. Amano, Nucl. Fusion 32, 633 (1992).

[4] K. Yamazaki et al., Fusion Eng. Des. 81, 2743 (2006).

[5] T. S. Hahm and K. H. Burrell, Phys. Plasmas 2, 1648 (1995).

[6] P. Zhou, W. Horton and H. Sugama, Phys. Plasmas 6, 2503 (1999).

[7] D. R. Ernst et al., Phys. Plasmas 7, 615 (2000).

[8] Y. B. Kim, P. H. Diamond and R. J. Groebner, Phys. Fluids B 3, 2050 (1991).

[9] F. L. Hinton and G. M. Staebler, Phys. Fluids B 5, 1281 (1993).

[10] B. Esposito et al., Plasma Phys. Control. Fusion 45, 933 (2003).

[11] L. R. Baylor et al., Nucl. Fusion 37, 445 (1997).

[12] P. B. Parks and R. J. Turnbull, Phys. Fluids 21, 1735 (1978). 